\title{
Nonlinear Vibration Control by Semi-active Piezo-actuator Damping*
}

\author{
Du WANG**, Zhongyang GUO*** \\ and Ichiro HAGIWARA****
}

\begin{abstract}
A flexible beam model with bonded piezo-patches is taken as the study object. In order to suppress the disturbance vibration of the beam, the imposed voltage to the bonded piezoelectric elements is controlled. After the theoretical analysis for piezoelectric patches and beam structure, a bilinear form of the state space equation is derived. In this electromechanically coupled model, the imposed voltage control is related to the beam deformation state. Therefore, it is possible to realize the proposed semi-active control through a low cost approach. The concept of semi-active control comes from the fact of that only the gain of the imposed voltage is adjusted and the voltage direction is reverse to the deformation sensing. As a result, the control voltage is controlled only in two-phase planes. Here, nonlinear state feedback control theory is applied to design a controller and the proposed control approach is testified by numerical simulation.
\end{abstract}

Key Words: Piezoelectricity, Bilinear Model, $H_{\infty}$ Control, Semi-active Control

\section{Introduction}

Advanced mechanical structures are required to have abilities to sense their own states, and furthermore to adjust themselves into optimal states. Therefore, it is imperative that there is a converter to transform structural states into accessible information, and also to convert a series of instruction into active driving forces exerted on structures to adjust their states. Piezoelectric elements are very suitable for such purposes, since they can generate electric charges, while experience deformation, and produce deformation in response to an active exciting voltage.

* Received 14th December, 2000

** Graduate School of Mechanical Engineering \& Science, Tokyo Institute of Technology, Ookayama 212-1, Meguro-ku, Tokyo 152-8552, Japan

*** School of Mechatronic Engineering, Harbin Institute of Technology, West Grand St.92, Harbin 150001, China

**** Department of Mechanical Sciences and Engineering, Tokyo Institute of Technology, Oolkayama 2-12-1, Meguro-ku, Tokyo 152-8552, Japan. E-mail: hagiwara@mech.titech.ac.jp
In the past decade, there has been tremendous interest in the use of piezoelectric sensors and actuators for structural control applications ${ }^{(1)-(4)}$. Piezoelectric elements have been successfully used in the active damping control and passive damping control for a variety of smart structures. In the active damping control mode, piezoelectric patches are attached to the surface of a structure at a position where a high level of strain is produced. Flexure, like bending, induces a voltage in the piezo-patches. The voltage is fed back to a controller for control calculation and then the calculated result signal is amplified to drive the piezo-patches. Hereafter, the piezo-patches translate the imposed voltage to produce deformation of anti-vibration which cancels the original disturbance. In the passive damping control mode, it requires no power amplifier. The piezo-patches detect vibration and convert it to a voltage that is sent to a resistive shunt circuit where it is dissipated by heat. In the active mode, the costly voltage amplifier is of high frequency response performance and the multi-mode vibration control can be realized. While in the passive mode ${ }^{(5),(6)}$, a simple circuit is needed but only the vibration of low frequency mode, e.g. 1st mode, could 
be controlled by the optimized circuit parameter. Here in this paper, a new approach in the vibration control by piezo-patches is proposed. The proposed method is a semi-active control mode for piezoactuator damping, where an operational amplifier that inverts sensing voltage $180^{\circ}$ out of its phase is used and its amplification ratio can be controlled. In the following, the contents are composed of the theoretic analysis, state space bilinear modeling for cantilever beam bonded with piezo-patches and nonlinear $H_{\infty}$ state feedback control design as well as numerical testification.

\section{Theoretical Basis for Piezoelectric Patches and Beam Structure}

The piezo-actuator is a part of structure and the stress or strain signal determined in the actuator can be processed in a control algorithm and then be fed to the actuator to control unwanted vibration in the structure.

The strain, electric field and electric displacement within a piezoelectric material can be fully described by a single pair of electromechanical equations and the best choice of equations depends on the problem. In the notation of IEEE Standard 176-1987, the following pair of equations is the equivalent statement of the electro-mechanical relationship. For the thin piezopatches, the stresses of $y$ and $z$ directions are ignored and only electric parameters $D_{3}$ and $E_{3}$ are considered. If the poling direction of piezoelectric patches is perpendicular to the surface of the patches, the constitutive relations of the patches are

$$
\left\{\begin{array}{l}
\varepsilon^{p}=d_{13} E_{3}+\sigma^{p} / Y^{p} \\
D_{3}=d_{13} Y^{p} \varepsilon^{p}+\left(\exists_{33}-d_{13}^{2} Y^{p}\right) E_{3}
\end{array} .\right.
$$

where $\sigma^{p}$ and $\varepsilon^{p}$ are the stress and strain normal to the poling direction and parallel to the direction of $x^{-}$ axis shown in Fig. $1, D_{3}$ and $E_{3}$ represent the electrical displacement and the electric field in the poling direction, and $d_{13}$, and $Y^{p}$ and $\ni_{33}$ designate the piezoelectric strain constant, Young's modulus, and the electric permittivity respectively. Certainly, the effect of the accumulation of electric charges on the piezoelectric capacitance can be calculated by integrating the electric displacement over the area of the patches. As shown in Fig. 1, two piezoelectric patches are bonded to the upper and lower surfaces of a beam. According to the constitutive relations (1) and the theoretic assumption of Bernoulli-Euler beams, the deformation compatibility, which requires the deformations of piezoelectric patches and the beam at their interface to be consistent, leads to

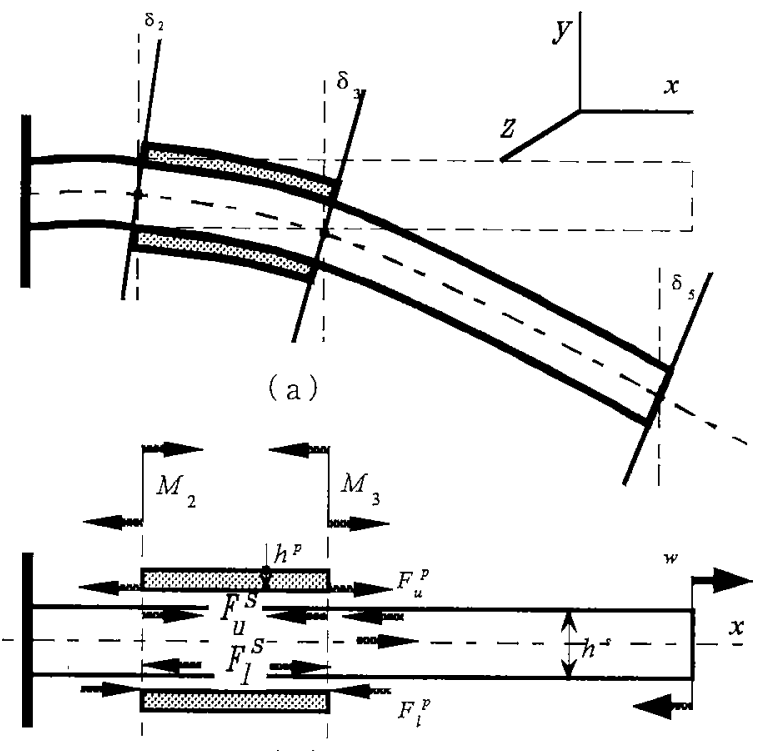

(b)

Fig. 1 Beam structure with bonded piezo- $0^{-}$atches

$$
\left\{\begin{array}{l}
\Lambda_{u}+\frac{F_{u}}{Y^{p} h^{p}}=-\frac{\left(h^{s}+h^{p}\right)}{4} \frac{\left(F_{u}-F_{l}\right) h^{s}}{Y^{s} I^{s}} \\
\Lambda_{l}+\frac{F_{l}}{Y^{p} h^{p}}=\frac{\left(h^{s}+h^{p}\right)}{4} \frac{\left(F_{u}-F_{l}\right) h^{s}}{Y^{s} I^{s}}
\end{array}\right.
$$

where,

$$
\left\{\begin{array}{l}
\Lambda_{u}=d_{13} E_{3}-\frac{1}{2}\left(1+\frac{h^{p}}{h^{s}}\right) \cdot\left(\varepsilon_{3}+\varepsilon_{2}\right) \\
\Lambda_{l}=d_{13} E_{3}+\frac{1}{2}\left(1+\frac{h^{p}}{h^{s}}\right) \cdot\left(\varepsilon_{3}+\varepsilon_{2}\right) \\
\left|F_{l}\right|=\left|F_{u}\right|=\left|F_{l}^{p}\right|=\left|F_{u}^{p}\right|=\left|F_{l}^{s}\right|=\left|F_{u}^{s}\right|
\end{array}\right.
$$

$Y^{s} I^{s}$ is the bending stiffness of the beam, $\varepsilon_{3}$ and $\varepsilon_{2}$ are the strains of the beam at their edges (the strain of the upper and lower piezoelectric patches are equal but in reverse sign). The superscripts $s$ and $p$ denote the substrate beam and the piezoelectric actuator respectively, while the subscripts $u$ and $l$ designate the upper and lower patches. Since the strains of the beam and piezoelectric patches at the interface are equal for ideally bonded patches, the active interaction forces $F_{u}$ and $F_{l}$ (force/width), as shown in Fig. 1 , are supposed existing only at both ends of the patches. It should be especially emphasized that $\varepsilon_{3}$ and $\varepsilon_{2}$ will be affected by external loads, the boundary conditions of the beam, the placement, dimensions, and properties of the piezoelectric patches, and even the active interaction forces; consequently, obtaining an explicit expression for them is not easy. However it is clear that they describe the strain of the beam, which need to be sensed and controlled.

The active interaction forces are related to the beam strain state $\varepsilon_{2}$ and $\varepsilon_{3}$ with the following expression. 


$$
\left\{\begin{array}{l}
\frac{F_{u}}{Y^{s} h^{s}}=\frac{1}{2(\phi+\alpha)}\left(1+\frac{h^{p}}{h^{s}}\right)\left(\varepsilon_{3}+\varepsilon_{2}\right) \\
\frac{F_{l}}{Y^{s} h^{s}}=-\frac{1}{2(\phi+\alpha)}\left(1+\frac{h^{p}}{h^{s}}\right)\left(\varepsilon_{3}+\varepsilon_{2}\right)
\end{array}\right.
$$

where

$$
\psi=\frac{Y^{s} h^{s}}{Y^{p} h^{p}} \quad \alpha=6\left(1+\frac{h^{p}}{h^{s}}\right) .
$$

To the pure bending beam, now we confine the discussion to the upper piezoelectric patch because the lower patch has the same formula except opposite sign. Then the definition of electrical capacitance is given out as:

$$
C^{p}=Q / V_{s}
$$

where $Q$ is the electric charge accumulated on the piezo patches and $V_{s}$ is the static circuit voltage imposed on the patches. The capacitance is composed of three parts, i.e., $C^{p}=C^{c}+C^{b}+C^{V}$. The first part is the capacitance of patch without deformation;

$$
C^{c}=\frac{S^{p}}{h^{p}}\left(\ni_{33}-d_{13}^{2} Y^{p}\right)
$$

The second part is the capacitance of the patch relaxed by its bonding to the elastic beam ;

$$
C^{b}=\frac{S^{p} d_{13}^{2} Y^{p}}{h^{p}}\left(1-\frac{\phi}{\psi+\alpha}\right)
$$

And the third part of the capacitance is that induced by the strain state of the beam;

$$
C^{V_{s}}=\frac{S^{p} d_{13}^{2} Y^{p}}{h^{p}}\left(\frac{\psi}{\psi+\alpha}\left(1+\frac{h^{p}}{h^{s}}\right) \cdot \frac{\varepsilon h^{p}}{d_{13} V_{s}}\right)
$$

Here, if the imposed voltage is specified, the variation of the capacitance demonstrates the change of the strain states. Therefore, the strain of the beam for the patched area can be sensed via measurement of the electrical capacitance. For the purely bending beam, the strain of patched area is written as

$$
\varepsilon=\frac{V_{s}}{S^{p} d_{13} Y^{p} h^{p}}\left(\frac{\phi+\alpha}{\phi\left(1+h^{p} / h^{s}\right)}\right) \cdot C^{v},
$$

where, $V_{s}, C^{V_{s}}$ and $\varepsilon$ can express the upper and lower beam variables only with reverse sign.

To obtain the part of the electrical capacitance only related to structural deformation, one should filter out the parts of Eq. (7), and ( 8 ). Thus, the self -sensing actuator technique based on a bridge circuit could be applied. As described above, it is clear that the interaction force can be controlled by an electric field while structural deformation can be sensed from the variation of the capacitance. In reference to Fig. 1 and 2, according to geometry deformation relationship, a simplified strain expression of the purely bent beam for the patched area can be written as

$$
\varepsilon=\frac{h^{s}}{2\left(l_{3}-l_{2}\right)}\left(\operatorname{tg} \delta_{3}-\operatorname{tg} \delta_{2}\right) \approx \frac{h^{s}}{2\left(l_{3}-l_{2}\right)}\left(\delta_{3}-\delta_{2}\right) .(11)
$$

Combing with Eq.(10), an asymptotic relation between the beam deformation state and induced voltage is established as

$$
\begin{aligned}
\varepsilon & =\frac{h^{s}}{2\left(l_{3}-l_{2}\right)}\left(\delta_{3}-\delta_{2}\right) \\
& =\frac{V_{s}}{S^{p} d_{13} Y^{p}}\left(\frac{\psi+\alpha}{\phi\left(1+h^{p} / h^{s}\right)}\right) \cdot C^{V_{s}} .
\end{aligned}
$$

Therefore, it is natural to understand that the deformation state of the bending beam can be controlled by imposing voltage reverse to the sensed voltage.

\section{Dynamic Modeling for Beam Bonded with Piezo-patches}

The beam parameters in Fig. 1 are shown in Tables 1 and 2. The modal parameters in Table 1 are referred to the result of modal experiment identification by Eigensystem Realization Algorithm. In the dynamic analysis for the piezo-patch bonded beam, 5 discrete modes are considered for the elastic beam. Hence, an explicit dynamic beam model for the discrete beam model with 5 modes is demonstrated in Fig. 2. The strain states caused by piezoelectric interaction (supposed acted at the patch edges) of $M_{2}$ and $M_{3}$ are written as

$$
\delta_{2}^{V}=\frac{M_{2} l_{2}}{Y^{s} I^{s}}, \quad \delta_{3}^{V}=\frac{M_{3} l_{3}}{Y^{s} I^{s}}
$$

\begin{tabular}{|c|c|c|c|}
\hline \multicolumn{2}{|c|}{$l \times b \times h^{s}$} & $30 \times 2.5 \times 3$ & $\mathrm{~cm}^{3}$ \\
\hline Young's modulus & $\overline{Y^{s}}$ & $1.93 \mathrm{E}+14$ & $\mathrm{~N} / \mathrm{m}^{2}$ \\
\hline poisson ratio & $\lambda^{s}$ & 0.3 & \\
\hline density & $p^{s}$ & $\begin{array}{l}8030 \\
\mathrm{~kg} / \mathrm{m}^{3}\end{array}$ & \\
\hline
\end{tabular}

Because of $M_{2}=-M_{3}$, then

$$
\delta_{2}^{V} l_{3}=-\delta_{3}^{V} l_{2}
$$

In order to control the bending vibration modes, imposed voltage $V(t)$ is varied according to the control instruction calculated from the bending state $\delta$.

Table 1 Discrete beam mode parameters

\begin{tabular}{|l|c|c|c|c|c|}
\hline item & 1 & 2 & 3 & 4 & 5 \\
\hline Mode:f(Hz) & 27.65 & 76.65 & 123.21 & 173.31 & 272.87 \\
\hline Mode: $\zeta$ & 0.0024 & 0.0051 & 0.0082 & 0.0117 & 0.0194 \\
\hline
\end{tabular}

Table 2 Beam parameters

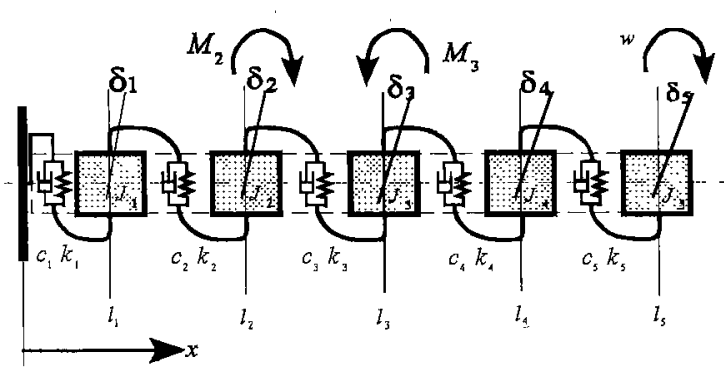

Fig. 2 Beam discrete model 
In the following step, the relationship between piezopatch area deformation of $\delta_{3}^{V}$ and $\delta_{2}^{V}$ which are caused by control voltage $V(t)$ and the actual beam bending state $\delta_{i},(i=1, \cdots, 5)$ is derived.

From Eq. (12) and (14), there are

$$
\frac{h^{s}}{2\left(l_{3}-l_{2}\right)}\left(1+\frac{l_{2}}{l_{3}}\right) \delta_{3}^{V}=\frac{V(t)}{S^{p} d_{13} Y^{p}}\left(\frac{\psi+\alpha}{\phi\left(1+h^{p} / h^{s}\right)}\right) \cdot C^{V(t)}
$$

and

$$
-\frac{h^{s}}{2\left(l_{3}-l_{2}\right)}\left(1+\frac{l_{3}}{l_{2}}\right) \delta_{2}^{V}=\frac{V(t)}{S^{p} d_{13} Y^{p}}\left(\frac{\phi+\alpha}{\psi\left(1+h^{p} / h^{s}\right)}\right) \cdot C^{V(t)} .
$$

By substituting Eq. (10) and (11) into (15) and (16), the following expressions can be obtained

$$
\frac{h^{s}}{2\left(l_{3}-l_{2}\right)}\left(1+\frac{l_{2}}{l_{3}}\right) \delta_{3}^{V}=\varepsilon \frac{V(t)}{V_{s}}=\frac{h^{s}}{2}\left(\delta_{3}-\delta_{2}\right) \frac{V(t)}{V_{s}}
$$

Then there exist,

$$
\left\{\begin{array}{l}
\delta_{3}^{V}=\frac{C^{V(t)} l_{3}\left(l_{3}-l_{2}\right)}{C^{V_{5}}\left(l_{3}+l_{2}\right)}\left(\delta_{3}-\delta_{2}\right) \cdot V(t) \\
\delta_{2}^{V}=-\frac{C^{V(t)} l_{2}\left(l_{3}-l_{2}\right)}{C^{V_{5}}\left(l_{3}+l_{2}\right)}\left(\delta_{3}-\delta_{2}\right) \cdot V(t)
\end{array} .\right.
$$

Therefore, by writing discrete dynamic equations of the beam bending vibration:

$$
\left\{\begin{array}{l}
J_{1} \ddot{\delta_{1}}+c_{1} \dot{\delta_{1}}+k_{1} \delta_{1}+c_{2}\left(\dot{\delta_{1}}-\dot{\delta_{2}}\right)+k_{2}\left(\delta_{1}-\delta_{2}\right)=0 \\
J_{2} \ddot{\hat{\delta}_{2}}-c_{2}\left(\dot{\delta_{1}}-\dot{\delta_{2}}\right)-k_{2}\left(\delta_{1}-\delta_{2}\right) \\
\quad+c_{3}\left(\dot{\delta_{2}}-\dot{\delta_{3}}\right)+k_{3}\left(\delta_{2}-\delta_{3}\right)+M_{2}=0 \\
J_{3} \ddot{\dot{\delta}_{3}}-c_{3}\left(\dot{\delta_{2}}-\dot{\delta_{3}}\right)-k_{3}\left(\delta_{2}-\dot{\delta}_{3}\right) \\
\quad+c_{4}\left(\dot{\delta_{3}}-\dot{\delta_{4}}\right)+k_{4}\left(\delta_{3}-\delta_{4}\right)-M_{3}=0 \\
J_{4} \ddot{\hat{\delta}_{4}}-c_{4}\left(\dot{\delta_{3}}-\dot{\delta_{4}}\right)-k_{4}\left(\delta_{3}-\delta_{4}\right) \\
\quad+c_{5}\left(\dot{\delta_{4}}-\dot{\delta_{5}}\right)+k_{5}\left(\delta_{4}-\dot{\delta}_{5}\right)=0 \\
J_{5} \ddot{\ddot{\delta}_{5}}-c_{5}\left(\dot{\delta_{4}}-\dot{\delta_{5}}\right)-k_{5}\left(\delta_{4}-\dot{\delta}_{5}\right)+w=0
\end{array}\right.
$$

From Eq. (18), the external action to the beam is related to the beam deformation state $\delta_{i}$ and control input $V(t)$, i.e.,

$$
M_{2}=-M_{3}=\underbrace{Y^{s} I^{s} \cdot \frac{C^{V(t)}}{C^{V_{5}}} \frac{l_{3}-l_{2}}{\left(l_{2}+l_{3}\right) V_{s}}}_{\xi} \cdot V(t) \cdot\left(\delta_{3}-\delta_{2}\right) .
$$

Suppose the output is charge $Q^{V}\left(Q^{c}, Q^{b}\right.$ could be filtered out by measurement calibration or bridge circuit), based on Eq. (6), (9), and (11) the derived result is

$$
y=Q^{V}=\underbrace{\frac{h^{s} S^{p} d_{13} Y^{p}}{2\left(l_{3}-l_{2}\right)} \cdot \frac{\psi}{\psi+\alpha}\left(1+\frac{h^{p}}{h^{s}}\right)}_{\mathcal{L}} \cdot\left(\delta_{3}-\delta_{2}\right)
$$

Hence, the bilinear form of the state space equations for the beam model with bonded piezo-patches are expressed as

$$
\left\{\begin{array}{l}
\dot{x}_{g}=A_{g} x_{g}+B_{w} w+B_{v} x_{g} u \\
y=C_{g} x_{g}
\end{array}\right.
$$

here

$$
x_{g}=\left[\begin{array}{llllllllll}
\delta_{1} & \delta_{2} & \delta_{3} & \delta_{4} & \delta_{5} & \dot{\delta_{1}} & \dot{\delta_{2}} & \dot{\delta_{3}} & \dot{\delta_{4}} & \dot{\delta_{5}}
\end{array}\right]^{T},
$$

$$
\begin{aligned}
& A_{g}=\left[\begin{array}{cc}
0_{5 \times 5} & I_{5 \times 5} \\
-K K / J J & -C C / J J
\end{array}\right] \\
& B_{w}=\left[\begin{array}{c}
0_{5 \times 1} \\
b_{w}
\end{array}\right] \quad B_{V}=\left[\begin{array}{cc}
0_{5 \times 5} & 0_{5 \times 5} \\
b_{V} & 0_{5 \times 5}
\end{array}\right] \\
& C_{g}=\left[\begin{array}{ll}
c_{g} & 0_{1 \times 5}
\end{array}\right] \text { and } u=V(t) \\
& J J=\operatorname{diag}\left[\begin{array}{lllll}
J_{1} & J_{2} & J_{3} & J_{4} & J_{5}
\end{array}\right] \text {, } \\
& K K=\left[\begin{array}{ccccc}
k_{1}+k_{2} & -k_{2} & 0 & 0 & 0 \\
-k_{2} & k_{2}+k_{3} & -k_{3} & 0 & 0 \\
0 & -k_{3} & k_{3}+k_{4} & -k_{4} & 0 \\
0 & 0 & -k_{4} & k_{4}+k_{5} & -k_{5} \\
0 & 0 & 0 & -k_{5} & k_{5}
\end{array}\right], \\
& C C=\left[\begin{array}{ccccc}
c_{1}+c_{2} & -c_{2} & 0 & 0 & 0 \\
-c_{2} & c_{2}+c_{3} & -c_{3} & 0 & 0 \\
0 & -c_{3} & c_{3}+c_{4} & -c_{4} & 0 \\
0 & 0 & -c_{4} & c_{4}+c_{5} & -c_{5} \\
0 & 0 & 0 & -c_{5} & c_{5}
\end{array}\right]
\end{aligned}
$$

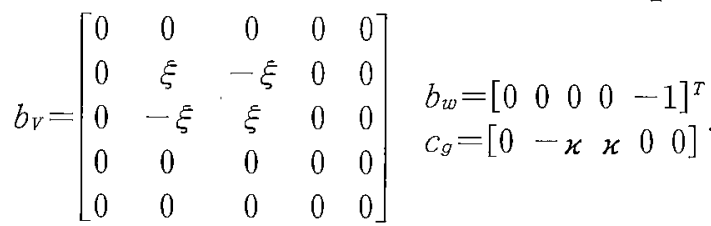

The above bilinear system (BLS) ${ }^{(7)}$ is not controllable at origin $x_{g}=0$. However, once the state $x_{g}$ leaves the origin, system becomes controllable. For the purpose of disturbance vibration control, it does not matter since $x_{g}=0$ means that the object has no displacement and velocity; that is to say, the object is not in vibration and need not to control either. Next, we will make use of nonlinear $H_{\infty}$ state feedback control law ${ }^{(8)}$ to design a controller for the above bilinear beam model.

\section{Controller Design with Semi-active Actucation}

Control voltage is stimulated by the beam strain state and its magnitude is proportionally changed with the strain state, i.e., $V(t) \propto \delta$. In order to pursue a low cost control method, here we are attempting to implement the control by adjusting the gain of voltage IC amplifier. In this way, because the control input voltage can only be produced in the accordance of strain state and the variant range is confined to the 2nd and 4th phase plane, this leads to the concept of semi-active control.

\section{1 Generalized plant}

To the bilinear system in Eq. (22), its generalized plant for control design is written as

$$
\left\{\begin{array}{l}
\dot{x}=A x+B_{1} w+B_{2} x u \\
z=\left[\begin{array}{l}
z_{1} \\
z_{2}
\end{array}\right]=\left[\begin{array}{l}
N_{1}(x) C_{11} \\
N_{2}(x) C_{12}
\end{array}\right] x+\left[\begin{array}{c}
0 \\
N_{2}(x) I
\end{array}\right] u .
\end{array}\right.
$$

where $x=\left[x_{g}^{T}, x_{1}^{T}, x_{2}^{T}\right]^{T} \in \boldsymbol{R}^{n}$ and $w \in \boldsymbol{R}^{m}, z \in \boldsymbol{R}^{p} \quad\left(z_{1} \in\right.$ $\left.\boldsymbol{R}^{p 1}, z_{2} \in \boldsymbol{R}^{p 2}\right) . \quad A, B_{1}, B_{2}, C_{11}, C_{12}$ are constant matrices. In Fig. $3, W_{1}(x)$ and $W_{2}(x)$ are proper robust weighting functions; and $N_{1}(x)$ and $N_{2}(x)$ are 
the nonlinear weighting functions. Because of the inclusion of nonlinear weighting functions, the evaluation output $z$ becomes nonlinear. In this case, the control performance can be evaluated by $L_{2}$ gain other than $H_{\infty}$ norm. At origin $x=0$, the bilinear system (23) is uncontrollable and its $L_{2}$ gain can not be improved. However, once the state $x$ leaves the origin, system becomes controllable and the control input $u$ can actively tighten the control performance index under the meaning of $L_{2}$ gain. This is very useful in taking the advantages of the properties of nonlinear actuator.

\section{2 Nonlinear $H_{\infty}$ state feedback control}

A sufficient condition for nonlinear $H_{\infty}$ state feedback control design is given as the following theorem.

Theorem 1: Given a possible small positive number $\gamma$ for the generalized plant (23). If there exists a positive definite matrix $\boldsymbol{P}_{i}$ which satisfies the following Algebraic Riccati Inequality (ARI),

$$
\boldsymbol{P} A+A^{T} \boldsymbol{P}+\frac{1}{\gamma^{2}} \boldsymbol{P} B_{1} B_{1}^{T} \boldsymbol{P}+C_{11}^{T} C_{11}+C_{12}^{T} C_{12}<0
$$

and the nonlinear weighting functions $N_{1}(x)$ and $N_{2}(x)$ satisfy the following condition

$$
\begin{gathered}
\left(\frac{1}{N_{2}^{2}(x)}-1\right) x^{T} \boldsymbol{P} B_{2} x\left(B_{2} x\right)^{T} \boldsymbol{P} x \\
\quad+\left(1-N_{1}^{2}(x)\right) x^{T} C_{11}^{T} C_{11} x \geq 0
\end{gathered}
$$

then a state feedback controller

$$
u(x)=-\left(\frac{1}{N_{2}^{2}(x)}\left(B_{2} x\right)^{T} \boldsymbol{P}+C_{12}\right) x
$$

can stabilize the system (23) with $L_{2}$ gain constraint $\|z\|_{2} \leq \gamma\|w\|_{2}$

In general, nonlinear weighting function $N_{1}(x)$ is designed for state $x$ and $N_{2}(x)$ is for control input $u$. The systematic design method for $N_{1}(x) \& N_{2}(x)$ is given in theorem 2 .

Theorem 2: For the generalized plant (23), the

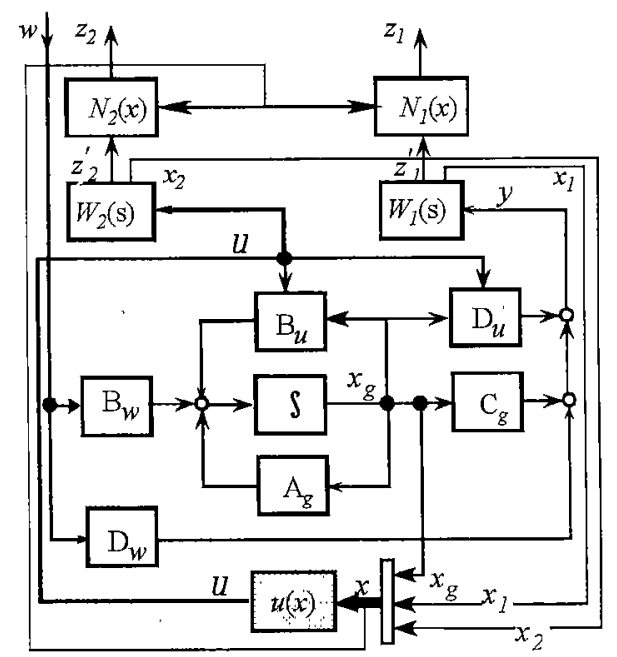

Fig. 3 Generalized control plant nonlinear weighting functions $N_{1}(x)$ and $N_{2}(x)$ that satisfy condition (25) are chosen as the followings.

$$
\begin{aligned}
& N_{1}(x)=\sqrt{1+N_{0}(x) x^{T} \boldsymbol{P} B_{2} x\left(B_{2} x\right)^{T} \boldsymbol{P} x} \\
& N_{2}(x)=\frac{1}{\sqrt{1+N_{0}(x) x^{T} C_{11}^{T} C_{11} x}}
\end{aligned}
$$

Here $\boldsymbol{P}$ is the solution of ARI (24) and $N_{0}(x)$ is selected as a positive scalar function of $x$.

Then the nonlinear $H_{\infty}$ state feedback controller is expressed as

$$
u(x)=-\left(1+N_{0}(x) x^{T} C_{11}^{T} C_{11} x\left(B_{2} x\right)^{T} \boldsymbol{P}+C_{12}\right) x .
$$

The discussion for the advantages of nonlinear $H_{\infty}$ control application is referred to reference ${ }^{(8)}$.

\section{3 Semi-active control discipline and concept implementation}

In the proposed control approach, the control input is produced by the amplification of the deformation feedback signal of piezoelectricity and only the control input (imposed voltage to piezoelectric patches) magnitude is adjusted according to deformation. Hence, it is obvious that the sensing voltage against the deformation strain is located in the first and third phase planes, while the control voltage is ranged in the second and fourth phase planes in order to recover the deformation (see Fig. 4). Then based on the above facts, the proposed methodology arises as, namely, piezoelectric semi-active damping (only two quarters of control input are available).

The control concept is going to be implemented as the sketch in Fig. 5. The semi-active control law is downloaded to DSP chip where the correspondent

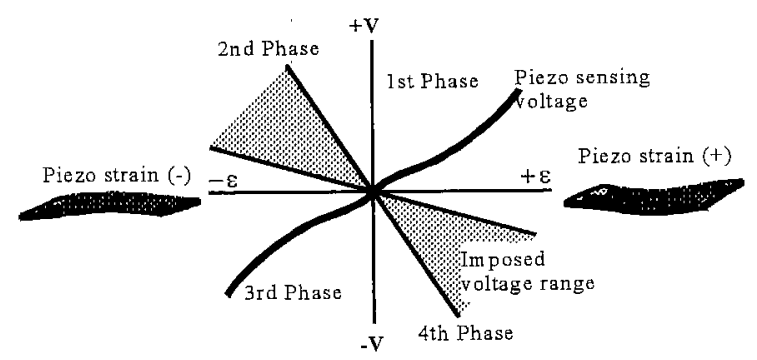

Fig. 4 Semi-active control discipline

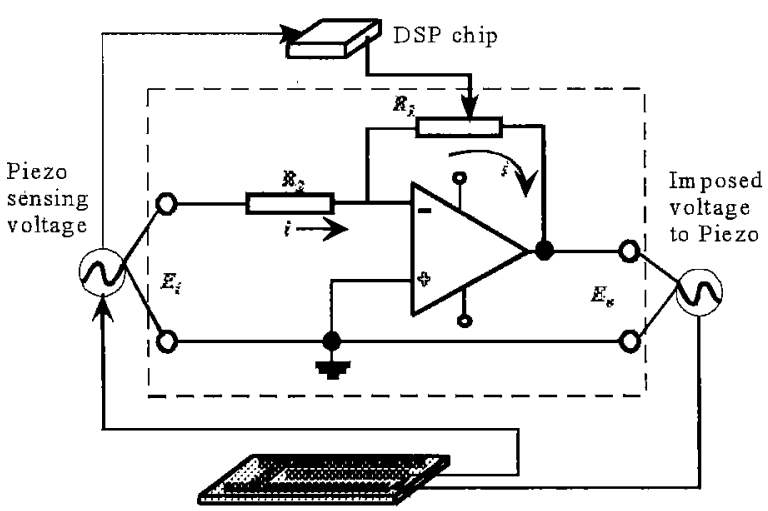

Fig. 5 Control implementation concept 
control voltage is calculated according to the deformation sensing voltage then the ratio of amplification is decided through adjusting related resistance $R_{1}$. In the practical design, the parameters in Fig. 5 should satisfy some conditions. Otherwise, the sensing voltage no longer represents the deformation strain rate but is contaminated by the control input voltage.

The relation between sensed voltage $E_{i}$ and piezo imposed voltage $E_{0}$ is expressed as

$$
E_{o}=-E_{i} \underbrace{R_{1}(t) / R_{2}}_{K_{a}} K_{r} .
$$

where $K_{a}$ is controllable amplification ratio and $K_{r}$ is amplification constant.

\section{Numerical Testification}

In order to demonstrate the proposed semi-active control approach, a numerical study is performed. The discrete beam model is taken as the 5 modes and its mode frequency and damping ratio are assumed as those values in Table 1 . In considering the control design, the augmented plant (Ref. Fig. 3) is that in Eq. (23). We select the weighting functions $W_{1}(s)$ and $W_{2}(s)$ based on the robust control design principle and they are notified in Eq. (31) and (32).

$$
\begin{aligned}
& W_{1}(s):\left\{\begin{array}{l}
\dot{x}_{1}=A_{w 1} x_{1}+B_{w 1} y \\
z_{1}^{\prime}=C_{w 1} x_{1}+D_{w 1} y
\end{array}\right. \\
& W_{2}(s):\left\{\begin{array}{l}
\dot{x}_{2}=A_{w 2} x_{2}+B_{w 2} u \\
z_{2}^{\prime}=C_{w 2} x_{2}+D_{w 2} u
\end{array}\right.
\end{aligned}
$$

Here the matrix components are listed as those in Eq. (33).

$$
\begin{aligned}
& A=\left[\begin{array}{ccc}
A_{g} & 0 & 0 \\
B_{w 1} C_{g} & A_{w 1} & 0 \\
0 & 0 & A_{w 2}
\end{array}\right], \\
& B_{1}=\left[\begin{array}{c}
B_{w} \\
B_{w 1} D_{w} \\
0
\end{array}\right], \quad B_{2} x=\left[\begin{array}{c}
B_{u} x_{g} \\
0 \\
B_{w 2}
\end{array}\right] \\
& C_{11}=\left[\begin{array}{lll}
D_{w 1} C_{g} & C_{w 1} & 0
\end{array}\right], \quad C_{12}=\left[\begin{array}{lll}
0 & 0 & C_{w 2}
\end{array}\right]
\end{aligned}
$$

\begin{tabular}{|c|c|}
\hline$l^{p} \times b^{p} \times h^{p}$ & $5 \times 2.5 \times 0.038 \mathrm{~cm}^{3}$ \\
\hline Young's modulus $Y^{p}$ & $6.8 \mathrm{E}+13$ \\
\hline poisson ratio & 0.31 \\
\hline density & $\mathrm{kg} / \mathrm{m}^{3}$ \\
\hline dielectric constant $\ni_{33}$ & $\begin{array}{l}635 \\
\text { Farad/m }\end{array}$ \\
\hline Piezo constant $\quad d_{13}$ & $\begin{array}{rc}-125 \times 10^{-12} & \mathrm{~m} / \mathrm{V} \\
& \text { or Coulomb/N }\end{array}$ \\
\hline Piezo-material coupling & \\
\hline Coefficient $\quad S^{p}$ & $\begin{array}{l}0.38 \\
\text { Coulomb/N/m }\end{array}$ \\
\hline
\end{tabular}

By applying the provided parameters in Tables 2 and 3 , then combining with the nonlinear $H_{\infty}$ controller calculated by Eq. (29), the simulation environment is

Table 3 Piezoelectric patch parameters

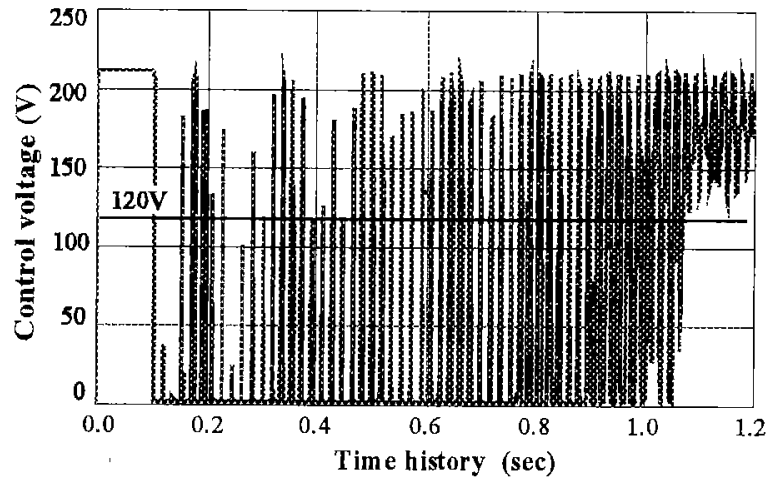

Fig. 6 Control voltage

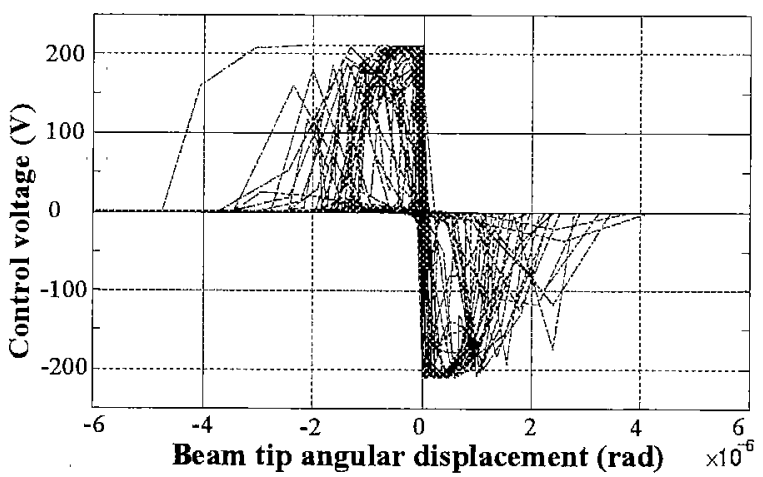

Fig. 7 Concurrent control voltage and beam deformation

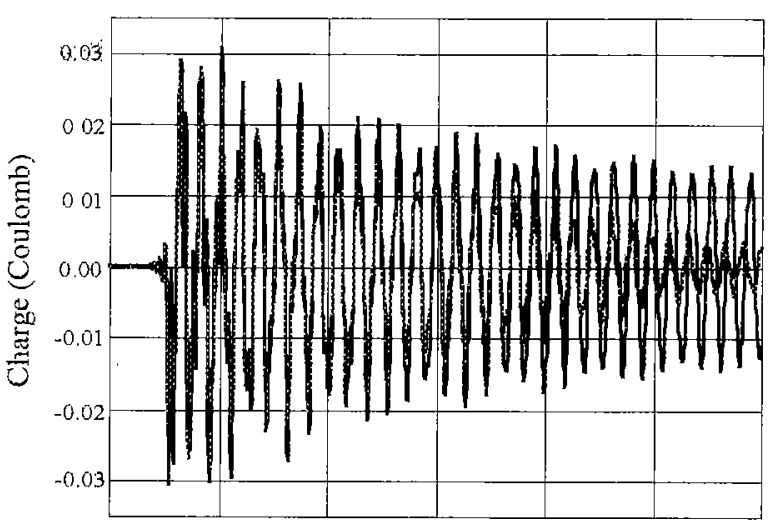

Time (sec)

Fig. 8 PZT Semi-active control (impulse disturbance)

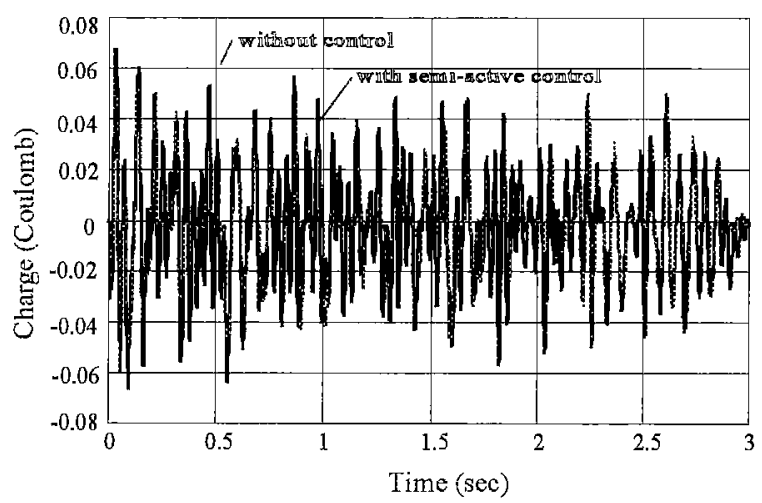

Fig. 9 PZT Semi-active control (random disturbance) 
completed. Thus, the controlled voltage is limited as the saturation bound $K_{a} \in[1.0,120]$ and operational amplification constant $K_{r}=3$. With an impulse disturbance input to the free end of the beam, the control voltage, shown in Fig. 6, is located in the 2nd and 4th phases in relation with beam deformation, see, Fig. 7. The control result for the charge variance of the piezo -patch is shown in Fig. 8. Moreover, the control result for a random disturbance to the beam free end is shown in Fig. 9. The charge variance expresses the beam deformation variance based on the theoretic formula. Therefore, the semi-active control for a beam bonded with piezo-patches is effective. In this simulation study, the deformation state of the discrete beam model is given out when the state feedback control algorithm is being calculated. For an actual control implementation, the systematic control layout can be the controller combined with an observer which is used to estimate the necessary state variables.

\section{Conclusions}

The semi-active control approach for piezopatch bonded beam is proposed and the feasibility is testified by simulation study. This control method can achieve almost the same effect as full active control system does but the system cost is lower at attempting to use an IC operational amplifier. The nonlinear $H_{\infty}$ state feedback control is applied in order to pursue a nonlinear control output for the imposing voltage. Though the nonlinear $H_{\infty}$ control is used in this study, the further investigation for control effectiveness with different control laws is expected in the near future and the future work will also be focused on the experimental testification and practical applications.

\section{References}

(1) Wang, Xiaoming, Ehlers, C. and Neitzel, M., An Analytical Investigation of Static Models of Piezoelectric Patches Attached to Beams and Plates, Smart Materials and Structure, No. 6 (1997), pp. 204-213.

(2) Yang, S.M. and Jeng, C.A., Structural Vibration Suppression by Concurrent Piezoelectric Sensor and Actuator, Smart Materials and Structure, No. 5 (1996), pp. 806-813.

(3) Dosch, J. and Inman D.J., A Self-Sensing Piezoelectric Actuator for Collocated Control, Journal of Intellectual Material System and Structure, Vol. 3, No. 1 (1992), pp. 166-185.

(4) Wang, Bor-Tsuen and Rogers, C.A., Modeling of Finite-Length Spatially-Distributed Induced Strain Actuators for Laminated Beams and Plates, Journal of Intellectual Material System and Structure, Vol. 2, No. 1 (1991), pp. 38-58.

(5) Ho, S.T., Matsuhisa, H. and Honda, Y., Passive Vibration Suppression of Beams by Piezoelectric Elements, Trans. Jpn. Soc. Mech. Eng., (in Japanese), Vol. 66, No. 643, C (2000), pp. 737-743.

(6) Ho, S.T., Matsuhisa, H. and Honda, Y., ActivePassive Hybrid Vibration Control of Beam by Self-Sensing Piezoelectric Actuator [the Optimal Resistance and Velocity Feedback], Trans. Jpn. Soc. Mech. Eng., (in Japanese), Vol. 66, No. 645, C (2000), pp. 1537-1543.

(7) Wand, Du, Guo, Z. and Hagiwara, I., Nonlinear Control Design for Polytopic Bilinear System, Proceedings of Dynamic \& Design Conference, Tokyo, JSME No. 00-6 (2000).

(8) Sampei, M., Nonlinear $H_{\infty}$ Control Theory and Its Application. SICE Seminar Lecture Note, (Robust Control Introduction), (in Japanese), (1999), pp. 87-110. 